\title{
The current and future landscape of radiotherapy for lung cancer
}

As guest editors, we are pleased to present this specially curated issue of Translational Lung Cancer Research, wherein we have invited leading experts from around the world to discuss currently evolving paradigms and controversies in the field of thoracic radiation oncology as we look to its future.

Radiotherapy for lung cancer was established as an important treatment modality in both the radical and palliative settings many years ago. Technologic advances in recent decades now afford an opportunity for this treatment to be more targeted than ever before in an era wherein the "precision" in precision medicine is a priority. While the delivery of more accurate forms of radiotherapy has minimized the risks of side-effects, numerous practical questions remain with regards to how best to utilize this treatment to optimize outcomes. For example, can stereotactic ablative radiotherapy (SABR), which is routinely prescribed and often low-risk, be safely delivered to moving lung tumors with an ultra-central location near the proximal bronchi? Might protons offer a clinical advantage for any or all stages of lung cancer? Do we currently have sufficient quality assurances in place as modern technologies are integrated into routine daily practice?

Beyond these technical questions, additional uncertainties have now arisen within the realm of modern multi-disciplinary care for lung cancer. Particularly as the rapid implementation of targeted and immunotherapies continues to shift the survival curves for patients with locally advanced and metastatic disease. Today, preliminary phase II data suggest that SABR might help many patients with oligometastatic stage IV non-small cell lung cancer (NSCLC) live longer; however, will that be the case for those receiving recently approved systemic therapies that weren't available when those trials were conducted only several years ago? Our invited experts provide an evidence-based review of these and other unresolved questions, including a focus on identifying the optimal role of radiotherapy for limited and extensive small cell lung cancer with regards to dose and fractionation, prophylactic cranial irradiation, and consolidative thoracic radiotherapy.

There is also a dedicated review that focuses on recent developments in palliative radiotherapy given the opportunity it offers to safely provide relief of uncontrolled symptoms whenever systemic therapies are no longer effective.

Finally, as we look to a future of lung cancer research that is arguably more exciting than ever before, we have to take a hard look at the role of comparative effectiveness studies that are frequently published. It is clear that randomized clinical trials cannot be completed for every clinical question, and therefore we have a dedicated article that addresses the inherent strengths and limitations of 'big data' science, which is being increasingly used to inform routine clinical practice.

We hope that all readers will enjoy this edition and learn as much, or even more, as we did from these authors who generously made the time to write these high-quality review papers for everyone to learn from.

\section{Acknowledgments}

None.

\section{Footnote}

Conflicts of Interest: The authors have no conflicts of interest to declare.

Ethical Statement: The authors are accountable for all aspects of the work in ensuring that questions related to the accuracy or integrity of any part of the work are appropriately investigated and resolved. 


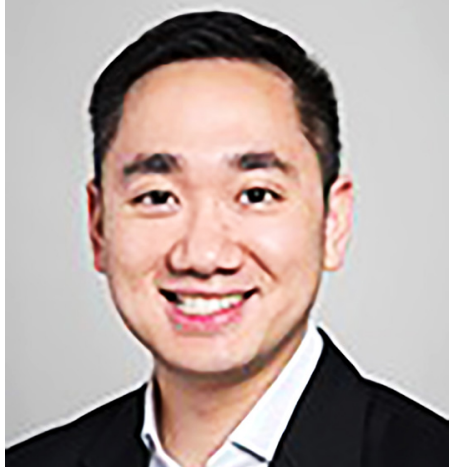

Alexander V. Louie

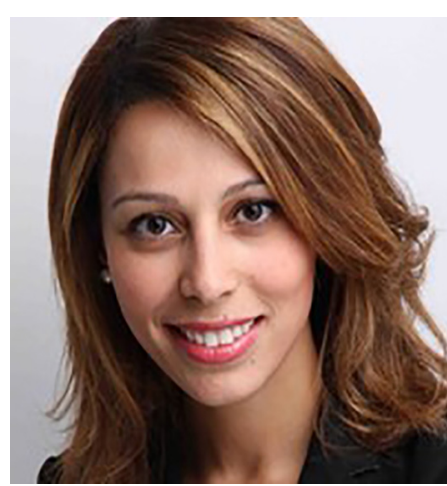

Houda Bahig

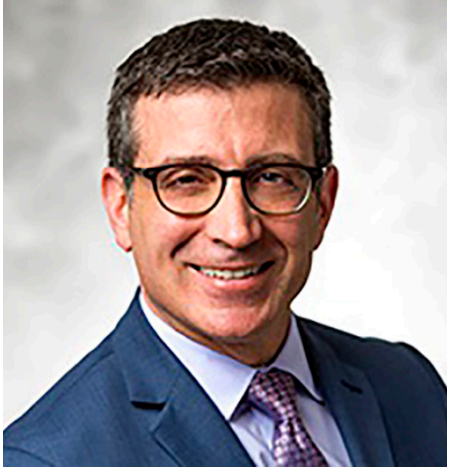

Drew Moghanaki

Alexander V. Louie ${ }^{1}$, MD, PhD, FRCPC ${ }^{1}$ Department of Radiation Oncology, Odette Cancer Centre-Sunnybrook Healtb Sciences Centre, Toronto, ON, Canada. (Email: dr:alexlouie@gmail.com)

Houda Bahig ${ }^{2}$, MD, PhD, FRCPC ${ }^{2}$ Department of Radiation Oncology, Centre Hospitalier de l'Université de Montréal, Montreal, QC, Canada.

(Email: bouda.babig.chum@ssss.gouv.qc.ca)

Drew Moghanaki ${ }^{3,4}$, MD, MPH

${ }^{3}$ Radiation Oncology Section, Atlanta Veterans Affairs Health Care System, Decatur, GA, USA;

${ }^{4}$ Winship Cancer Institute, Emory University, Decatur, GA, USA.

(Email: drew.moghanaki@va.gov)

doi: 10.21037/tlcr.2019.09.07

View this article at: http://dx.doi.org/10.21037/tlcr.2019.09.07

Cite this article as: Louie AV, Bahig H, Moghanaki D. The current and future landscape of radiotherapy for lung cancer. Transl Lung Cancer Res 2019;8(Suppl 2):S122-S123. doi: 10.21037/tlcr.2019.09.07 